\title{
Airway hyper-responsiveness and small airway function in children with well-controlled asthma
}

\author{
Jianfeng Huang ${ }^{1}$, Mingzhi Zhang' ${ }^{1}$ Xiaobo Zhang' and Libo Wang'
}

BACKGROUND: Airway hyper-responsiveness (AHR) and small airway function are critical to children with asthma. Little is known about the role of the small airway in well-controlled subjects with AHR. We aimed to evaluate AHR and small airway function in children with well-controlled asthma, and to investigate the association between them.

METHODS: We studied 116 cases of children with well-controlled asthma (group A), 46 cases healthy children as controls (group C). Spirometry, impulse oscillometry (IOS), and methacholine challenge test (MCT) were conducted on all the children.

RESULTS: (i) Group A and group C had no differences in forced expiratory volume in $1 \mathrm{~s}\left(\mathrm{FEV}_{1}\right)$ and $\mathrm{FEV}_{1} /$ forced vital capacity (FVC) ratio $(P>0.05)$. Forced expiratory flow between 25 and $75 \%$ of vital capacity $\left(\mathrm{FEF}_{25-75}\right)$ and reactance at $5 \mathrm{~Hz}$ (X5) in group A were significantly lower than those in group C. (ii) One hundred and five cases (90.5\%) of group A proved positive to MCT. (iii) $\mathrm{FEF}_{25-75}$ in group A proved positive to MCT but were lower than those proved negative $(P<0.05)$.

CONCLUSION: AHR persisted in majority of children with well-controlled asthma. Among children with well-controlled asthma, small airway function was lower in those with AHR than those without AHR.

A sthma is the most common chronic lower respiratory disease in childhood throughout the world. The newest Global Initiative for Asthma guidelines, as well as international consensus on pediatric asthma suggested that, after initial severity assessment, ongoing care should include continual assessment of the patient's asthma control in terms of real-life activities $(1,2)$. This approach uses a cycle of detailed assessment and intervention to achieve and maintain asthma control. The level of control is defined by frequency of asthma symptoms, and the degree of lung function (forced expiratory volume in $1 \mathrm{~s}\left(\mathrm{FEV}_{1}\right)$, peak expiratory flow). But in some cases called "well controlled asthma" with no disease activity and normal lung function, how can we evaluate and manage them? Airway hyper-responsiveness (AHR) is a clinical feature of asthma and is often in proportion to the underlying severity of the disease. AHR of children with well-controlled asthma can display condition of the disease. But, the evaluation of AHR is not popular in pediatric clinic. In areas without this means, how can we do next?

Asthma is an inflammatory airway disorder that involves also the small airways (defined as the peripheral membranous bronchioles $<2 \mathrm{~mm}$ in diameter) (3). Two decades ago, Wagner et al. (4) demonstrated a more than sevenfold increase in small airway resistance in asymptomatic asthma with normal spirometry $\left(\mathrm{FEV}_{1}\right.$ and forced vital capacity (FVC)). The contribution of the small airways to the development of the clinical features and functional impairment in asthma is incompletely understood (5). Study suggested that asthmatics with AHR have more severe small airways obstruction, but little is known about the role of the small airways in asymptomatic subjects with AHR. Therefore, the aim of our study was to evaluate AHR and small airway function in children with well-controlled asthma (group A) compared to healthy control (group C), and to investigate the association between them.

\section{RESULTS}

The participants in this study were 116 children with well-controlled asthma ( 80 boys and 36 girls), 46 age-matched control subjects ( 30 boys and 16 girls). Proportion of family history of atopy or asthma, eczema, and allergic rhinitis were more in group A than in group C (Table 1 ).

All participants underwent spirometric tests, impulse oscillometry (IOS), and methacholine inhalation challenge safely. There were no significant differences in FVC, $\mathrm{FEV}_{1}$, $\mathrm{FEV}_{1} / \mathrm{FVC}$, resistance at $5 \mathrm{~Hz}$ (R5), R5 -R20 between two groups. Forced expiratory flow between 25 and $75 \%$ of vital capacity $\left(\mathrm{FEF}_{25-75}\right)$ of predicted and reactance at $5 \mathrm{~Hz}$ (X5) in group A were significantly lower than those in group $\mathrm{C}$ (Table 2).

One hundred and five of 116 in group A proved positive to methacholine challenge, and 12 of 46 in healthy group C. Results of methacholine challenge test (MCT) had significant differences between the two groups (Figure 1).

$\mathrm{FEF}_{25-75}$ in group A displayed positive results to methacholine challenge and were significantly lower than those in the same group displayed negative results to methacholine 
challenge. We did not detect analogous difference in the results obtained by IOS in group A (Table 3 ).

\section{DISCUSSION}

Asthma is the most common chronic lower respiratory disease in childhood throughout the world. Therefore, appropriate asthma management may have a major impact on the quality of life of patients and their families, as well as on public health outcomes. Recent international guidelines for the assessment and monitoring of asthma have defined the concepts of asthma control. Asthma control is the degree to which manifestations of the disease are reduced or removed by therapy. Asthma control is not intrinsic to the patient and can fluctuate over time. Control categories are quite relevant in clinical practice. By appropriate asthma management, we can achieve better control. Subsequent management in children with well-controlled asthma is also an important proposition to pediatrician $(1,2)$.

Children with well-controlled asthma have no current impairment (symptoms, need for rescue medication, limitation of activities), and future risk (lung function, no exacerbations, no medication side effects). They are asymptomatic with normal spirometry $\left(\mathrm{FEV}_{1}\right.$ and FVC). Therefore, the development of reliable and noninvasive methods to assess well-controlled asthma in children remains a priority and is essential for the effective management of asthma, for instance, guide to step down inhaled corticosteroids. Muñoz et al. (6)

Table 1. Baseline characteristics of children with well-controlled asthma (group A) and healthy controls (group C)

\begin{tabular}{lcccc}
\hline & $\begin{array}{c}\text { Group A } \\
(N=116)\end{array}$ & $\begin{array}{c}\text { Group C } \\
(N=46)\end{array}$ & $P$ value & $t / \chi^{2}$ \\
\hline Age (year) & $8.19 \pm 2.10$ & $8.71 \pm 2.74$ & $P>0.05$ & -1.27 \\
Male/female & $80 / 36$ & $30 / 16$ & $P>0.05$ & 0.02 \\
Height $(\mathrm{cm})$ & $129.2(121,141)$ & $137.5(120,150)$ & $P>0.05$ & -1.515 \\
Weight $(\mathrm{kg})$ & $29(24,35)$ & $32(27,38)$ & $P>0.05$ & -1.953 \\
Family history of & $54 / 62$ & $4 / 42$ & $P<0.05$ & 18.92 \\
atopy or asthma & & & & \\
$\begin{array}{l}\text { Parental smoking } \\
\text { Eczema }\end{array}$ & $34 / 82$ & $8 / 38$ & $P>0.05$ & 1.85 \\
$\begin{array}{l}\text { Allergic rhinitis } \\
\text { Current inhaled }\end{array}$ & $68 / 48$ & $7 / 39$ & $P<0.05$ & 23.24 \\
corticosteroids & 16 & $5 / 41$ & $P<0.05$ & 30.46 \\
$\begin{array}{l}\text { Current } \\
\text { montelukast }\end{array}$ & 0 & 0 & - & - \\
\hline
\end{tabular}

demonstrated that airway inflammation persisted in most well-controlled asthma patients despite the fact that their condition was controlled, and therefore, measurement of bronchial inflammation seems essential to achieve proper asthma control. Stojković-Andjelković et al. (7) evaluated AHR in 106 children randomly selected from general pediatric population. The prevalence rate of AHR was $18 \%$. Among the asthmatic children who did not show asthma symptoms or did show mild asthma symptoms, bronchial sensitivity, one aspect of AHR, remained unchanged after 2 y of treatment by Global Initiative for Asthma These results are in line with our study (105 of 116 asymptomatic subjects in group A remained AHR). AHR is a condition of excessive airway irritability and can be a prognostic marker of asthma persistency and severity, and is used to monitor long-term treatment effects. AHR is reported to decrease through childhood (8). During our research, we detected that the majority of well-controlled asthma expressed AHR. AHR persists in children with wellcontrolled asthma, which means airway inflammation exists; so, subsequent treatment is required. We should incorporate MCT into management of children with well-controlled asthma in the future, and should survey the recovery period of AHR with different levels.

Asthma is an inflammatory disease of the entire airway. The pathophysiology of asthma involves inflammatory and structural changes that have, for the most part, been described in the large airways (9). Due to technical considerations, it has been considerably easier to sample the large airways in human asthmatic patients, for example, by induced sputum to assess

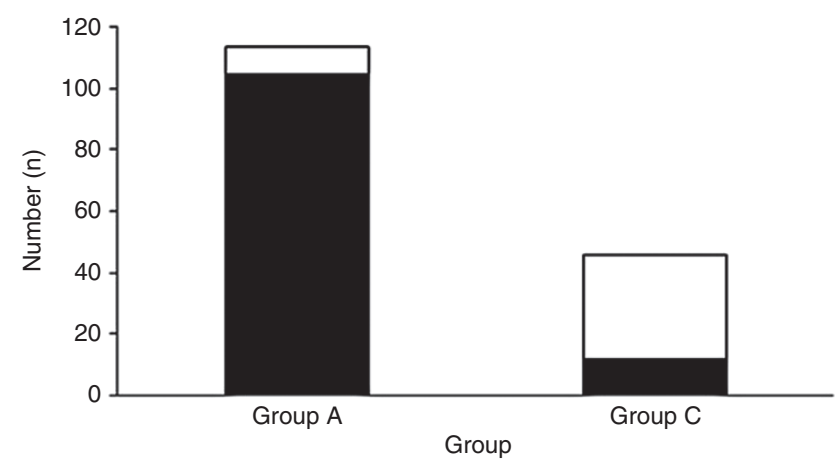

Figure 1. Comparison of methacholine challenge test (MCT) results between children with well-controlled asthma (group A) and healthy controls (group C). One hundred and five of 116 in group A proved positive to methacholine challenge, and 12 of 46 in healthy group C. Results of methacholine challenge had significant differences between the two groups $(P<0.05)$. $\mathbf{\square}$, Positive to MCT; $\square$, Negative to MCT.

Table 2. Summary of spirometry and impulse oscillometry results for children with well-controlled asthma (group A) and healthy controls (group C)

\begin{tabular}{lccccccc}
\hline & $\mathrm{FVC}(\%)$ & $\mathrm{FEV}_{1}(\%)$ & $\mathrm{FEV}_{1} / \mathrm{FVC}$ & $\mathrm{FEF}_{25-75}(\%)$ & $\mathrm{R} 5(\mathrm{KPa} / \mathrm{l} / \mathrm{s})$ & $\mathrm{R} 5-\mathrm{R} 20(\mathrm{KPa} / \mathrm{l} / \mathrm{s})$ & $\mathrm{X} 5(\mathrm{KPa} / \mathrm{l} / \mathrm{s})$ \\
\hline Group A & $98.81 \pm 8.46$ & $102.97 \pm 10.92$ & $87.15 \pm 6.12$ & $88.56 \pm 20.94$ & $0.39(0.27,0.58)$ & $0.07(0.03,0.15)$ & $-0.21(-0.32,-0.16)$ \\
Group C & $95.52 \pm 12.05$ & $104.74 \pm 16.08$ & $89.03 \pm 4.88$ & $99.13 \pm 22.67$ & $0.51 \pm 0.23$ & $0.11 \pm 0.09$ & $-0.16 \pm 0.1$ \\
$t / z$ & 1.83 & -0.75 & -1.86 & -2.61 & -1.58 & -1.20 & -4.40 \\
$P$ value & 0.068 & 0.455 & 0.065 & 0.01 & 0.112 & 0.23 & $P<0.001$ \\
\hline
\end{tabular}

FEV , forced expiratory volume in $1 \mathrm{~s}$; FVC, forced vital capacity. 
Table 3. Summary of spirometry and impulse oscillometry results for children with well controlled asthma (Group A) distinguished by MCT

\begin{tabular}{lccccccc}
\hline & $\mathrm{FVC}(\%)$ & $\mathrm{FEV}_{1}(\%)$ & $\mathrm{FEV}_{1} / \mathrm{FVC}$ & $\mathrm{FEF}_{25-75}(\%)$ & $\mathrm{R} 5(\mathrm{KPa} / \mathrm{l} / \mathrm{s})$ & $\mathrm{R} 5-\mathrm{R} 20(\mathrm{KPa} / \mathrm{l} / \mathrm{s})$ & $\mathrm{X} 5(\mathrm{KPa} / \mathrm{l} / \mathrm{s})$ \\
\hline MCT negative & $97.92 \pm 10.12$ & $102.5 \pm 12.11$ & $88.45 \pm 4.95$ & $95.12 \pm 20.8$ & $0.46 \pm 0.18$ & $0.11 \pm 0.09$ & $-0.25 \pm 0.09$ \\
MCT positive & $99.38 \pm 7.74$ & $103.15 \pm 10.51$ & $86.66 \pm 6.46$ & $87.31 \pm 21.73$ & $0.35(0.27,0.56)$ & $0.07(0.03,0.11)$ & $-0.2(-0.3,-0.16)$ \\
$t / z$ & 1.03 & 0.28 & -1.37 & -2.04 & 0.932 & 0.954 & 1.11 \\
$P$ value & 0.309 & 0.78 & 0.172 & 0.043 & 0.35 & 0.322 & 0.17 \\
\hline
\end{tabular}

FEV , forced expiratory volume in $1 \mathrm{~s}$; FVC, forced vital capacity; $\mathrm{MCT}$, methacholine challenge test.

inflammation and endobronchial biopsy to evaluate structural remodeling. However, a number of studies investigating lung tissue from autopsy and surgical resections have indicated that eosinophilic inflammation also occurs, often to a greater degree, in the small airways of asthmatic patients. Originally referred to as the "silent zone", small airway may not be that silent after all. Important clinical correlates are asthma exacerbations and airways remodeling, exercise asthma, and nocturnal asthma (10). Thus, to control pathology in the small airways has become a desirable goal in asthma management. It has become increasingly clear that one important reason for this lack of disease control can be explained by persistent uncontrolled inflammation in the small airways (11-13). Asthma patients with no symptoms, normal lung function, can also find obstruction in small airway. $\mathrm{FEF}_{25-75}$ is generally considered as an approximate measure of the small airways caliber and thus its reduction represents a small airways obstruction caused by asthma inflammation (14). $\mathrm{FEF}_{25-75}$ percent predicted should be evaluated in clinical studies of asthma in children and might be of use in predicting the presence of clinically relevant reversible airflow obstruction. In our study, $\mathrm{FEF}_{25-75}$ had significant differences between the two groups. Although there are no recommendations regarding the utility of the percent predicted $\mathrm{FEF}_{25-75}$ by the American Thoracic Society or the National Asthma Education and Prevention Program (NAEPP), this measurement may have clinical significance in managing childhood asthma. Simon et al. (15) suggested that $\mathrm{FEF}_{25-75}$ percent predicted was well correlated with bronchodilator responsiveness in asthmatic children with normal FEV1. In our study, $\mathrm{FEF}_{25-75}$ of children with well-controlled asthma proved AHR but were lower than those without AHR $(P<0.05)$. Therefore, we think that $\mathrm{FEF}_{25-75}$ can be used to evaluate the AHR of well-controlled asthma. Specially in area without MCT, the test of small airway function can provide help for the control of asymptomatic asthma. We think it is also the first study to reveal the association between AHR and small airway function in children with well-controlled asthma. The inflammation and dysfunction in small airway count for the persistent AHR; so, we will focus on the importance and potential clinical benefit of targeting the small airway in treatment of children with well-controlled asthma in the future.

The IOS system has been developed as a patient-friendly lung function test that minimizes demands on the patient and requires only passive cooperation of the subject wearing a nose clip, keeping lips tightly closed about a mouthpiece and breathing normally through the mouth. IOS has been shown to be useful in the diagnosis of asthma and small airway impairment in children. However, studies on the utility of IOS to assess children with well-controlled asthma are limited, and there are few published for IOS measurements among those subjects with or without hyper-responsiveness. Shi et al. (13) assessed asthma severity and control in 101 asthmatic patients, 6-17 y of age in a cross-sectional study. They demonstrate that the controlled asthma group and healthy controls have no differences in small airway parameters tested by IOS. In contrast, other study (16) and our study have shown that the parameters reflected small airway function were statistically different between children with well-controlled asthma and healthy children. However, in our study, we did not detect the difference in results obtained by IOS in subgroups of group A with or without hyper-responsiveness. Following explanations may account for the potential reason: (i) We assessed AHR by Dmin; however, some other studies measured AHR by spirometry. Therefore, unified criterion should be established to measure AHR in the future. (ii) Age is an important factor affecting lung function and AHR. Large-sample study is needed to perform comparisons in different stage of age. Further studies will also be needed to compare the results in different pediatric asthma phenotypes.

In our study, we assessed well-controlled asthmatics with small airway dysfunction by parameters of lung function. On morphology, airway remodeling is an essential component of asthma, which is associated with fixed airflow limitation. There are two important aspects of asthma assessment. The limitation of our study was the deficiency of parameter-reflected airway remodeling. We would consider airway remodeling in the future.

In conclusion, children with well-controlled asthma are asymptomatic, but the majority of well-controlled asthma in our study expressed AHR. Among children with wellcontrolled asthma, small airway function was lower in those with AHR than those without AHR. Difference of small airway function gives clues to diagnose and manage these patients. Specially, in area without bronchial challenge test, the test of small airway function can provide help for the control of asthma.

\section{METHODS \\ Subjects}

Study subjects (group A). The subjects of this study were children who visited the Children's Hospital of FuDan University from September 2012 to September 2013 with asthma. Diagnosis of asthma and treatment were based on the Global Initiative for Asthma guidelines. Definition of well-controlled asthma was described as no symptoms of asthma day or night, no need to use rescue medication, no limitation of activities, normal spirometry $\left(\mathrm{FEV}_{1}\right.$ and $\left.\mathrm{FVC}\right)$, no 
exacerbation, and no medication side effects, at least $12 \mathrm{mo}$, based on the Global Initiative for Asthma (2).

Control subjects (group C). Control subjects were age-matched healthy children free of chronic respiratory and atopic diseases. No child in the control group was receiving medication. Written informed consent and assent were obtained from all participants and their parents or guardians.

All subjects gave informed consent before participating. The study was approved by the Research Ethics Committee of Children's Hospital of FuDan University. The clinical data do not disclose the identity of the subjects under the study.

\section{Measurements}

Spirometry. Spirometry was performed using master screen electronic spirometer (Jaeger, Wezburg, Germany). The system was calibrated every morning using a $1-\mathrm{L}$ syringe and recalibrated at least every $4 \mathrm{~h}$. Integrated volumes were corrected for body temperature, ambient pressure and saturated with water vapor. Spirometry was performed in accordance with the American Thoracic Society guidelines. $\mathrm{FEV}_{1}, \mathrm{FVC}$, and forced expiratory flow between 25 and $75 \%$ of vital capacity $\left(\mathrm{FEF}_{25-75}\right)$ were reported as \% predicted and FEV1/FVC ratio $\left(\mathrm{FEV}_{1} / \mathrm{FVC}\right)$ as raw values only.

IOS. IOS was performed using masterscreen electronic spirometer (Jaeger, Wezburg, Germany). IOS was used to measure the resistance at $5 \mathrm{~Hz}(\mathrm{R} 5)$, which reflects total airway resistance, the resistance at $20 \mathrm{~Hz}$ (R20), which reflects the resistance in the large airways, and the difference between R5 and R20(R5-R20), reflecting the resistance in the small airways. In addition, reactance at $5 \mathrm{~Hz}$ (X5) was measured, which reflects elastic properties of the small airways.

Methacholine inhalation challenge. Methacholine inhalation challenges were performed by Astograph aerosol generator (J-21; Chest Company; Tokyo, Japan) delivered increasing doses of methacholine from 12 serially arranged nebulizers (17). Dmin was the cumulative dose of methacholine at the inflection point of the respiratory resistance (Rrs) tracing. One Dmin unit was considered to be equal to $1 \mathrm{~min}$ of inhaling an aerosolized methacholine solution of $1.0 \mathrm{mg} / \mathrm{ml}$ during tidal breathing. Conventionally, AHR was judged as Dmin $<7.0 \mathrm{U}(18)$.

Statistical analysis. The statistical analyses were performed with the IBM SPSS Statistics, version 20 (IBM, Armonk, NY). Descriptive statistics were computed as mean and SD for continuous variables, or median and 25th-75th percentiles in case of skewed distribution, and as absolute frequency and percent for categorical variables. Absolute frequencies were compared using $\chi^{2}$ test. The Mann-Whitney test was used for non-normally distributed continuous variables and Student's $t$-test for normally distributed continuous variables.

\section{STATEMENT OF FINANCIAL SUPPORT}

No financial assistance was received to support this study.

Disclosures: No conflicts of interest in connection with this article.

\section{REFERENCES}

1. Papadopoulos NG, Arakawa $\mathrm{H}$, Carlsen $\mathrm{KH}$, et al. International consensus on (ICON) pediatric asthma. Allergy 2012;67:976-97.

2. Global Initiative for Asthma (GINA). Pocket guide for asthma management and prevention, 2014. http://www.ginasthma.org/local/uploads/files/ GINA_Pocket_2014_Jun11.pdf.

3. van der Wiel E, ten Hacken NH, Postma DS, van den Berge M. Smallairways dysfunction associates with respiratory symptoms and clinical features of asthma: a systematic review. J Allergy Clin Immunol 2013;131:646-57.

4. Wagner EM, Liu MC, Weinmann GG, Permutt S, Bleecker ER. Peripheral lung resistance in normal and asthmatic subjects. Am Rev Respir Dis 1990;141:584-8.

5. Malagrinò L, Catapano G, Novelli F, et al. Markers of small airway involvement and asthma control in patients with moderate-to-severe asthma. Ann Allergy Asthma Immunol 2014;112:551-2.

6. Muñoz X, Sanchez-Vidaurre S, Roca O, Torres F, Morell F, Cruz MJ. Bronchial inflammation and hyperresponsiveness in well controlled asthma. Clin Exp Allergy 2012;42:1321-8.

7. Stojković-Andjelković A, Obradović S, Vuletić B, Radlović N. Change of bronchial hyperresponsiveness in asthmatic children. Srp Arh Celok Lek 2011;139:316-21.

8. Riiser A, Hovland V, Mowinckel P, Carlsen KH, Carlsen KL. Bronchial hyperresponsiveness decreases through childhood. Respir Med 2012;106:215-22.

9. Johnson JR, Hamid Q. Appraising the small airways in asthma. Curr Opin Pulm Med 2012;18:23-8.

10. Bjermer L. The role of small airway disease in asthma. Curr Opin Pulm Med 2014;20:23-30.

11. Keen C, Olin AC, Wennergren G, Gustafsson P. Small airway function, exhaled $\mathrm{NO}$ and airway hyper-responsiveness in paediatric asthma. Respir Med 2011;105:1476-84.

12. Husemann K, Haidl P, Kroegel C, Voshaar T, Kohlhäufl M. [Lung function diagnostics for the small airways]. Pneumologie 2012;66:283-9.

13. Shi Y, Aledia AS, Tatavoosian AV, Vijayalakshmi S, Galant SP, George SC. Relating small airways to asthma control by using impulse oscillometry in children. J Allergy Clin Immunol 2012;129:671-8.

14. Sposato B, Scalese M, Migliorini MG, Di Tomassi M, Scala R. Small airway impairment and bronchial hyperresponsiveness in asthma onset. Allergy Asthma Immunol Res 2014;6:242-51.

15. Simon MR, Chinchilli VM, Phillips BR, et al.; Childhood Asthma Research and Education Network of the National Heart, Lung, and Blood Institute. Forced expiratory flow between $25 \%$ and $75 \%$ of vital capacity and FEV1/ forced vital capacity ratio in relation to clinical and physiological parameters in asthmatic children with normal FEV1 values. J Allergy Clin Immunol 2010;126:527-34.e1-8.

16. Song TW, Kim KW, Kim ES, Park JW, Sohn MH, Kim KE. Utility of impulse oscillometry in young children with asthma. Pediatr Allergy Immunol 2008;19:763-8.

17. Takami S, Mochizuki H, Muramatsu R, Hagiwara S, Arakawa H. Relationship between bronchial hyperresponsiveness and lung function in children age 5 and 6 with and without asthma. Respirology 2013;18:682-7.

18. Hiroyuki M, Hirokazu A, Kenichi T et al. Bronchial sensitivity and bronchial reactivity in children with cough variant asthma. Chest 2005;128:2423-7. 